\title{
ADAPTIVE FINITE ELEMENT METHODS FOR CONTINUUM DAMAGE MODELING
}

\author{
J. B. Min, $†$ W. W. Tworzydlo, $\ddagger$ and K. E. XIques \\ †NASA/Marshall Spece Flight Center, Huntsville, AL 35812, U.S.A. \\ †Computational Mochanics Company, Austin, TX 78752, U.S.A. \\ \$Adaptive Rescarch Corporation, Huntsville, AL 35805, U.S.A.
}

(Received 9 August 1994)

\begin{abstract}
The paper presents an application of adaptive finite element methods to the modeling of low-cycle continuum damage and life prediction of high-temperature components. The major objective is to provide automated and scourate modeling of damaged zones through adaptive mesh refinement and adaptive time-stepping methods. The damage modeling methodology is implemented in an wrual way by embedding damnge evolution in the tranxient nonlinear solution of elasto-viscoplastic deformation problems. This nonlinear boundary-value problem is diecretized by adaptive finite element methods. The automated h-adaptioe meah refinements are driven by error indicators, bused on selected principal variables in the problem (streases, nonelastic string, damare, etc.). In the time domain, adaptive timestepping is used, combined with a predictor-corrector time marching alporithm. The time atep selection is controlled by required time sccuracy. In onder to take into socount strong temperature dependency of material parametern, the nonlinear structural solution is coupled with thermal amalyes (one-way coupling). Several test examples illustrate the importance and benefits of adaptive meah refinements in accurate prediction of damage levels and failure time.
\end{abstract}

\section{INTRODUCTION}

Unified viscoplastic constitutive models have evolved over the last 20 years to provide a means for analytically representing a material response from the elastic through the plastic range, including strain-rate dependent plastic flow, creep and stress relaxation. These theories are guided by physical considerations including dislocation dynamics and are based on the principles of continuum mechanics.

The first multi-dimensional formulations of elastoviscoplastic constitutive equations was due to Bodner and Partom [1]. Since then, a number of constitutive models have appeared; many of these theories are summarized in review articles that appear in Ref. [2]. A NASA-Lewis Research Center sponsored research program (HOST) $[3,4]$ conducted by the Southweat Research Institute recently concluded a four year research effort to develop unified constitutive models for isotropic materials and to demonstrate their usefulness for analysis of high temperature gas turbine engines. One result of this study is material property data for high temperature nickel-based alloys over a wide temperature range. The unified models employed were those of Bodner-Partom and Walker. More recently, a viscoplastic constitutive model was developed at NASA Lewis by Freed [I] and Freed and Verilli [6, and then refined by Freed and Walker [7]. Relevant material constants were determined experimentally for aluminum, copper and nickel.
Unified viscoplastic theories have been inplemented by a number of finite element researchers. The first use of unified constitutive modek in a finite element program was by Newman et al. [8]. Later, under the NASA HOST program, the Bodner-Partom and Walker models were implemented into finite element codes by General Electric and Pratt and Whitney. Kaufman et al. [9] describes these efforts as applied to gas turbine components. Moreno and Jordan [10], under the NASA HOST program, developed and applied a unified constitutive model to gas turbine combustors using the MARC code. Recently, a finite element viscoplastic study of cylindrical thrust chambers was presented by Arya [11] Detailed studies of several rate-dependent plasticity models and their numerical implementation using adaptive finite element methods were conducted by Bass and Oden [12]. These studies were extendod for thermoviscoplastic analysis by Thornton el al. [13]

Models of material damage and retiability studies are relatively recent developments, but significant progress has been made in understanding and modeling these problems. The models of continumm damage development, which are of primary interest here, are based on micro-mechanical studies of micro-crack nucleation and growth, discussed by Achby and Raj [14], Greenwood [15] and many other researchers. Phenomenological descriptions of these models are usually based on the carly work of Kachanov [16], where the general evolution law was introduced for 


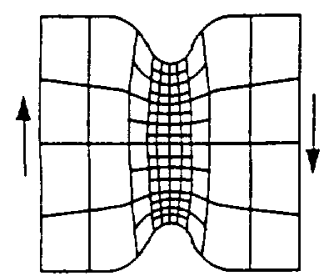

(a)

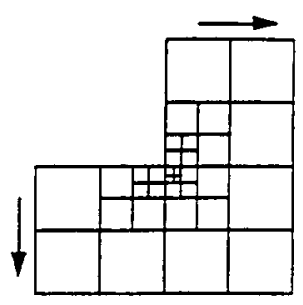

(b)
Fig. 1. Adaptive mesh refinements for damage modeling. (a) resolution of distributed damage zones. (b) localization of macro-crack initiation.

damage parameter. These, models fit very well into the framework of unified viscoplastic theories, and several detailed evolution forms have been proposed (see, for example, papers of Bodner and Chan [17], Lockie and Hayhurst [18], Simo [19], and references therein). The equations presented in these works are convenient for the description of low-cycle damage, where the damage occurs relatively quickly under a general stress history. For problems with cyclic loads, the evolution of damage is more conveniently expressed in terms of the number of cycles. Theories of this type were reviewed by Nelson [20]. In highcycle fatigue modeling, they are computationally more efficient than time integration methods. To date, no adaptive finite element solutions have been reported for continuum damage modeling in the literature.

Therefore, the main objective of this study was to prove the feasibility and practical benefits of using adaptive finite element methods to model continuum damage and to improve life prediction capabilities for high-temperature components and other structures subjected to hostile thermo-mechanical environments.

The major benefit of such an application of adaptive methods is that the adaptive mesh refinement based on rigorous error estimators, can provide automated and exact resolution of critical damage zones (Fig. 1a) and, moreover, an automated indication of possible indication of macto-cracts (Fig. lb). Independent of adaptive mesh refinement, an adaptive time-stepping method can provide automated control of the accuracy of time-marching procedures, and greatly minimine the computational effort necessary to obtain a final solution.

\section{UNIFTED VISCOPLASTIC THEORIES AND CONTINUUM DAMAGE MODELINGS}

Complex mechanical behavior of many hightemperature engineering materials can be improved by the constitutive models known as unified elastoviscoplastic theories. These theories, consistent with micro-mechanics of metallic materials, describe in a unified manner various aspects of elastic and nonelastic behavior of structures. More, these approaches are readily expandable to include such effects as crack nucleation, continuum damage, etc. In this study the Bodner-Partom $[1,17]$ and the Freed-Walker constitutive theories [ $5-7]$ were implemented for damage modeling. It should be noted that presently there exists a wide variety of viscoplastic constitutive theories and corresponding damage models. However, the primary objective of this paper is not to study these models, but to develop and demonstrate adaptive finite element techniques in application to damage modeling. Hence the above two constitutive theories were implemented in this study. Importantly, the adaptive finite element tochniques developed herein is applicable to a much wider class of continuum damage models.

\subsection{Basic formulations}

The internal-state-variable constitutive formulations are to be used in the solution of infinitesimal, quasistatic, isothermal, initial-boundary-value problems. Consider a viscoplastic structure occupying a region $\mathbf{Q}$ with boundary $\partial \mathbf{Q}$. The behavior of the structure is described by the following system of differential equations:

(1) Equilibrium in rate form:

$$
\dot{\sigma}_{W}+b_{1}=0
$$

where $\sigma_{y}$ denote components of the stress tensor, $b_{i}$ are the body force components per unit volume, and the summation convention is employed.

(2) Kinematic relation for velocity gradients:

$$
\epsilon_{y}=\epsilon_{y}^{\mathrm{z}}+\epsilon_{\nu}^{p}=\left(\dot{u}_{w}+\dot{u}_{p_{1}}\right) / 2
$$

where $\epsilon_{y}$ denotes the total strain components and superscripts $E$ and $P$ denote elastic and inelastic strain components, respectively. The components of the displacement rates are $\dot{u}_{4}$.

(3) Conatitutive relations:

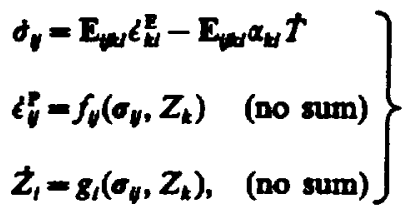

where $\mathbf{E}_{\text {w }}$ represent Hooke's tensor of elasticity parameters, $\alpha_{t t}$ are components of a tensor of thermal expansion parameters, and $T$ represents the rate of the change in temperature. Both $\mathbf{E}_{\boldsymbol{u} \text { s }}$ and $\alpha_{t}$ are temperature dependent. The constitutive functions are $f_{V}$ and $g_{i}$ where $Z_{k}$ represent internal state variables. These functions and state variables characterize the viscoplastic response of the material.

The description of the problem is completed by prescribing the boundary and initial conditions, 


$$
\left.\begin{array}{c}
\dot{u}=\dot{y}_{1} \text { on } \partial \Omega_{1} \\
\delta_{y} n_{j}=\delta_{1} \text { on } \partial \Omega_{2},
\end{array}\right\}
$$

where $\partial \Omega=\partial \Omega_{1} \cup \varphi \Omega_{2}, \hat{H}_{1}$ are prescribed surface displacement rates, the components of a unit normal vector, and $b$, the prescribed surface traction rates. The initial conditions include specifying the displacements, stresses, and internal state variables, i.e.

$$
u_{l}(x, 0), \sigma_{\sigma}(x, 0), Z_{l}(x, 0) \quad x \in \Omega
$$

\subsection{Continnum damage models}

The continuum damage modeling describes the deterioration of mechanical properties of materials by development of intergrain cracks and voids. According to material ecience theories presented, for example, by Ashby and Raj [14] or Greenwood [15], the growth of damage at grain boundaries is expresced in terms of two mechanisms: nucleation and growth. The damage parameter $\boldsymbol{\omega}$ is then introduced, and can be interpreted as the ratio of damaged cross section ( $\left.A_{\text {rod }}\right)$ (or, alternatively, volume) to the original area $\left(A_{\text {mominel }}\right)$ :

$$
\infty=\frac{A_{\text {wid }}}{A_{\text {moined }}}
$$

The micro-cracks considered here are in the range of $0.01 \mathrm{~mm}$ in length. The rupture criterion is $\omega=1$, which corresponds to the saturation of the material with voids. Alternatively, a single crack may grow to a size on the order of $1 \mathrm{~mm}$. In the latter case, the crack is too big to be treated in a continuum sense, and its propagation should be followed using the methods of fracture mechanics.

Continuum damage modeling is easily incorporated into the viscoplastic constitutive theories by introducing the evolution law for the damage parameter. Usually the general Kachanov form based on separation of variables is accepted:

$$
\omega=f_{1}(\omega) f_{2}(\sigma)
$$

where $\sigma$ is the stress ate in the body.

More generally, other parameters can be used in the evolution law. Moreover, the damage parameter is often decomposed into isotropic and anisotropic components. Within this class, two low-cycle damage models have been considered in this study based on the Bodner and Chan [17] and Greenwood [15] models. However, there are several problems with practical application of directional damage, reliability of these models and conducting experiments relevant for the evolution of necessary parameters. Even the extensive experiments presented in Refs. $[3,4,17]$ did not provide all the necessary data and, hence, the damage model was limited to the isotropic damage. Therefore, numerical implementation of this study was also limited to isotropic damage.
Once the damage parameter $\omega$ is calculated, it should be somehow incorporated into the constitutive theory. This is based on a straight forward interpretation of the damage parameter, $\omega$, as a ratio of area of voids in a given cross-section to the original area, as shown in eqn (6). The result of this reduction of area is that the real stress $\left(\sigma_{\mathrm{m}}\right)$ in a material is higher than the nominal stress $\left(\sigma_{\mathrm{mo}}\right)$ :

$$
\begin{aligned}
& \sigma_{\mathrm{mal}}=\frac{A_{\text {monim }}}{A_{\text {mad }}} \sigma_{\text {mom }} \\
& =\frac{A_{\text {monimal }}}{A_{\text {monim }}-A_{\text {mod }}} \sigma_{\text {mom }} \\
& =\frac{\sigma_{\operatorname{mon}}}{(1-\infty)} \text {. }
\end{aligned}
$$

In the Bodner-Partom theory this results in the modification of the nonelastic strain rate equation. The details of this modification can be found in/Ref. [17]. The same principle, applied to Freed-Walker theory [7], leads to the use of a real stress rather than a nominal stress in the evolution equation. Therefore, to incorporate continuum damage, the stress deviator $\left(S_{\psi}\right)$ in the viscoplastic model used in this study is being replaced by:

$$
S_{y} \stackrel{\longrightarrow}{\longrightarrow}(1-\omega) S_{v}
$$

This replacement means that the atresses stored in the program are nominal stresses, but the nonelastic fow rule operates on real stresses. Consequently, the back stress $B$ and the drag stress $D$ in their model are real stresees.

\section{FTNTE IIMMTNT SOLUTION OF VISCOPLASTIC PROALEMS WTH DAMAGE}

The boundary-value problem described in the previous sections is strongly nonlinear and can only be solved by numerical techniques, such as the finite element method. Application of this method requires recasting of the problem in a weak, variational form.

\subsection{Weak formulation}

The rate form of the equilibrium equations described earlier can be viewed as a constraint condition on the constitutive equations. A weak formulation of the equilibrium equations, sufficient to include the necessary constraint condition, is developed here. The equilibrium conditions in terms of the strain rates are of the form

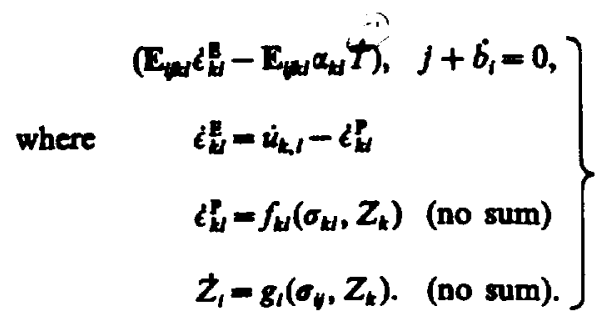


Here

$$
\left.\begin{array}{l}
\sigma_{v}=\int_{0}^{t} \dot{\sigma}_{y} \mathrm{~d} t+\dot{\theta}_{y} \\
\dot{\sigma}_{v}=\mathrm{E}_{v t} \dot{\epsilon}_{k t}^{\mathrm{E}} \\
Z_{i}=\int_{0}^{t} Z_{i} \mathrm{~d} t+\tilde{Z}_{i}^{\prime}
\end{array}\right\}
$$

where $\sigma_{v}$ and $Z_{l}$ are understood to depend upon $u_{i J}$ and to satisfy initial conditions.

In order to obtain a weak formulation of a boundary-value problem the space of displacement rates (solution space of trial functions) is introduced.

$$
U=\left\{\dot{u} \in\left[W^{m,}(\Omega)\right\}^{N}, \dot{i}(x) \rightarrow 0 \text { as }\|x\| \rightarrow \infty\right\}
$$

and the space of test functions

$$
V=\left\{v \in\left[W^{m},(\Omega)^{N}, \quad r(x) \rightarrow 0 \text { as }\|x\| \rightarrow \infty\right\}\right.
$$

where $\boldsymbol{\Omega}$ is a computational domain, $N$ is the dimension of the physical space (2 or 3), and $W^{m}$. $(\Omega)$ is the Sobolev space, where specific values of $m, p$ and $q$ depend on the particular forms of constitutive equations governing the material under consideration.

Multiplying the equilibrium equation by a suitably smooth test function $v_{1}=v_{1}(x)$ and integrating over $\boldsymbol{\Omega}$, the weak form of the rate equilibrium equations can be obtained:

$$
\int_{0} \Phi_{i \omega} v_{i} d \Omega=0 \text { for all } v_{i} \text { in } V \text {. }
$$

After the substitution of the constitutive equations, application of the divergence theorem and a grouping of terms the following variational problem is obtainod:

Find a displacement rate field $t \rightarrow \dot{\mathrm{i}}(\mathrm{x}, t)$ in $V+\{\bar{u}\}$ such that for every $t$

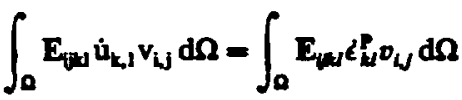

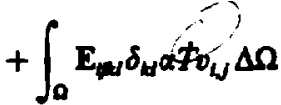

$$
\begin{aligned}
& +\int_{0} \dot{b}_{1} b_{1} \mathrm{~d} \Omega+\int_{a_{2}} i_{i} v_{i} \mathrm{~d} s
\end{aligned}
$$

for all $v_{i}$ in $V$.

The rates of nonelastic strains $\epsilon_{k l}$ are obtained from the relevant constitutive theory, and the pointwise values of rates of temperature $T$ were read in from the file created by thermal analysis program. Here $\mathrm{d \Omega}$ and ds are volume and surface measures, $\overline{\boldsymbol{n}}$ is any function defined on $\Omega$ such that its trace on the boundary segment $\partial \Omega_{1}$ is (where $\dot{u}_{1} \partial \Omega_{1}-u_{1}$ ). It is easily verified that any sufficiently smooth solution of eqn (1) will also satisfy the governing equations.
Conversely, any solution of the governing equations and boundary conditions will also satisfy eqn (15).

\subsection{Finite element formulation}

A finite element approximation of eqn (15) is obtained by introducing approximations of the trial and test functions:

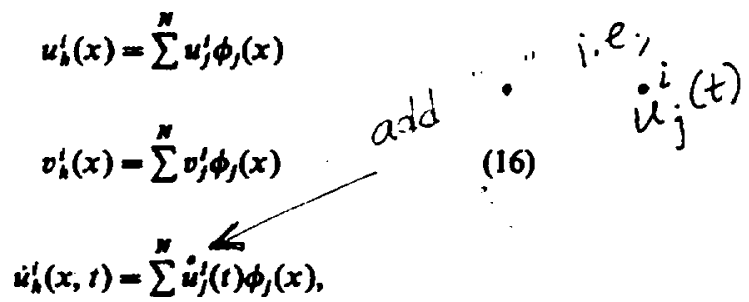

where $N$ is the number of nodes, $i$ indicates the vector components, $\phi_{j}$ is a basis function, and $u_{j}^{\prime}, f_{j}^{\prime}$ and $\dot{u}_{j}^{\prime}(t)$ are the values of $u_{h}^{l}, v_{l}^{l}$, and $\dot{u}_{h}^{l}(t)$ at node $j$ at time $t$, respectively.

Recasting these approximations into a matrix form and employing standard notation, gives the time element approximation for $\dot{\mathrm{v}}(x, t)$ at time $t$

$$
\begin{aligned}
\int_{a} B^{T} E B d a & =\int_{a} B^{T} E^{p} d \Omega+\int_{a} B^{T} E \alpha d \Omega \\
& +\int_{a} \phi^{T} b d \Omega+\int_{a_{z}} \phi^{T} d s,
\end{aligned}
$$

where $\boldsymbol{\Omega}$ denotes the element volume, $\partial \boldsymbol{\Omega}_{\mathbf{2}}$ denotes an element surface where tractions are defined, and $B$ is the strain displacement matrix.

The temperature affects the viscoplastic structura] analysis directly in three ways: (1) the elasticity matrix $\Sigma(T)$ and the coefficient of thermal expansion $\alpha(T)$ depend on temperature, (2) nodal loads- $F(T)$ depend on the local temperature rates $(T)$, and (3) several parameters in the viscoplastic constitutive model are temperature dependent.

\subsection{Viscoplastic solution method with damage madeling}

In this section, an algorithm used for the solution of viscoplastic problems with damage modeling is presented. In addition to this basic algorithm, an interface was provided for the thermal analysis, wherein temperature histories can be read into the structural code.

An uncoupled (sometimes called one-way coupled) formulatoin was used for this approach, wherein the thermal problem is solved first, followed by the viscoplastic analysis. The transient thermal problem is solved by time marching with a time step $\Delta t_{T}$, and the nodal temperatures at arcessive times $t_{1}, t_{2}, \ldots$. are obtained. These temperature vectors are used as input to the structural analysis.

The first computation in the structural analysis is to solve an initial statics problem if the initial temperature distribution $T(x, 0)$ is not equal to a uniform 
reference temperature or if any initial static loads are present. The results of this analyzis are the initial conditions (displacements and streses) for the transient viscoplastic analysis.

The viscoplastic analysis time marches with a time step $\Delta t_{3}$. Experience has shown that the time step required for the structural analyai is usually smaller than for the thermal analysis, i.e. $\Delta T_{0}<\Delta t_{T}$. At intermediate times in the structural andy is, the temperatures are linearly interpolated from the temperatures known at the beginning and end of the brger thermal time intervals.

A computationally efficient method for tracing the solution of initial-boundary-value problems in viscoplasticity, which utilize a hidden variable constitutive formulation is described here. The strategy employed in the viscoplastic algorithm is a follows:

With the initial distribution of strese, temperature, and internal variable specified, we the equilibrium equations to obtain the nodal deplacement rates. Then integrate the constitutive equations forward in time at the element Gauss integration points. With updated values of the stress, temperature, and internal variables at the new time, the equitibrium equations are solved again. This sequence is determining the nodal displacement rates, then advancing the constitutive equations in time is contineod mntil the desired history of the initial-boundary-value problem has been obtained. Thus, the algorithm proceeds through the following steps:

(1) At time $t_{n}=0$, initialize $\sigma_{y}, Z_{7}$ and $\omega_{1}$ for each element.

(2) Calculate $\epsilon_{y}^{p}=f_{y}\left(\sigma_{y}, Z_{k}, \infty_{1}\right)$ for each element at $t=t_{n}$ [thus determining the right-hand side of eqn (17)].

(3) Assemble and solve the equitirium condition for $a_{n}\left(t_{n}\right)$.

(4) Calculate $\varepsilon_{y}\left(t_{n}\right)=\mathrm{B} \dot{u}_{n}\left(t_{n}\right)$ for each element.

(5) Calculate $\dot{\phi}_{p}\left(t_{n}\right)=\mathbf{E}\left(\dot{\epsilon}_{y}\left(t_{n}\right)-\epsilon_{f}^{*}\left(t_{n}\right)\right)-\operatorname{Eo} T\left(t_{n}\right)$.

(6) Calculate $Z_{1}\left(t_{n}\right)=g_{1}\left(\sigma_{v}\left(t_{m}\right), Z_{t}\left(t_{n}\right)\right)$.

(7) Calculate $\dot{\omega}_{i}\left(t_{n}\right)=f_{i}\left(\omega_{1}\left(t_{n}\right), \sigma_{n}\left(t_{n}\right)\right)$.

(8) Integrate $\phi_{y}, z_{i}$ and $\omega_{i}$ formurd over some appropriate $\Delta t_{1}$ for each element to get

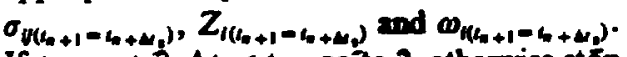

(9) If $t_{n+1}=t_{n} 7 \Delta t_{1}<t_{\text {tan }}$ go to 2, otherwise stóp.

Step 8 characterizes an explicit echeme, and this step can be replaced by a subroutine for implicit method if needed. If predictor-corrector type integration schemes are used, a slight modification is necessary beginning with Step 8 to be described in Section 4.2. The computational wathod above has been presented for a constant time step $\Lambda_{2}$. The computational experience by several invetigators $[9,12$, $16,21]$ indicates that a very $\sin$ il time step can be required because of the mathematicaly stifi nature of the constitutive equations describing the internal state variables. To gain improved efficiency and reliability, a variable time step algorithm has been implemented in this study. This technique will be described in the next section.

\section{ADAPTTVE COMPUTATTONAL TBCHNRQUDS}

In this study, adaptive computational techniques were used to provide the maximum reliability of results at the minimum computational cost. These techniques are used to adapt their parameters to changing characteristic of the solution during the solution process. In this study, two basic adaptive methods were used, namely $h$-adaptioe mesh refinement and adaptive time stepping. The basics, of these tochniques will be discussed on the following subections.

\subsection{Error estimation and adaptive mesh refinement}

Adaptive mesh refinement, based on rigorous error estimates, enables to resolve a basic question of modern computational mechanics: what is the accuracy of the numerical solution? equivalently, how good are the answers? To systematically reduce error by adaptively changing mesh aizes or spectral orders of approximations, one must obviously have some means to judge the distribution of error in a numerical solution.

Some of the most important results related to error extimation and adaptive techniques for finite element methods and details of their derivations and proofs can be found in Refs. [22-24]. The error estimators and indicator are the basis for adaptive mesh refinements, which modify the mesh in onder to minimize the error at minimal computational cost. In this study, interpolation error estimators combined with $h$-refinement sechrigues have been implemented for the solution of clasto-viscoplastic problems with damage. The interpolation methods use the interpolation theory of finite elements in Sobolev norms to produce rapid (sometimes crude) estimates of the local error over individual elements. Simplifying this somewhat gives an error eatimate defined as the maximom difierence of the effective strain within the union of that particular element $\boldsymbol{Q}_{\mathbf{a}}$ and its neighboring elements. The $h$-methods are the mesh refinement methods, based on local subdivision of elements into emaller elements. An $h$-type refinement strategy may be outlined as follows [11]:

(1) Given a solution at time $t^{n}$, solve the initialboundary-value problem until time $t^{*+1}$ and calculate the estimated error for each element. The interval $t^{n+1}-t^{n}$ is much larger than the time marching step $\Delta t$.

(2) Determine the magnitude of the bryest elemental error $E_{\text {max }}$, and set a threshold value for refinement $\alpha E_{\text {max }}$, where $\alpha$ is a fraction between $x=0$ and one.

(3) Determine the number of elements to be refined using the threshold value. If this number of elements is greater than $\beta N_{\text {}}$ where $N_{0}$ is the number of elements and $\beta$ is a fraction between ano and one, a uniform distribution of error exists and no refinement 
is made (go to Step 1). Otherwise, refine all elements for which $E_{k}>\alpha E_{\max }$, where $E_{\mathrm{k}}$ is the error for a typical element $k$ in the mesh.

(4) If refinement has occurred, go back to time $t^{\prime \prime}$, interpolate the solution on a new mesh, and resolve the boundary value problem on the new mesh. Then again estimate the elemental error at time $t^{n+1}$.

(5) If element errors exist such that $E_{k}>\propto E_{\text {go }}$ to Step 3. Otherwise the errors have been reduced sufficiently, go to Step 1 with a new refined mesh, and new starting time $t^{n+1}$.

It is quite essential for the accuracy of the solution that after mesh refinement at time $z^{n+1}$ the solution process is backed to the time $t^{n}$. This assured that the interval $t^{n} \rightarrow t^{n+1}$ is covered on adequately refinod mesh, so there is no excessive accumulation of error.

\subsection{Adaptive time stepping techniques}

Time-dependent problems are usually solved by time marching techniques, where the time domain is discretized into a number of steps $t_{1}, t_{2}, \ldots, t_{n}$, and the solution proceeds by solution of a sequence of incremental problems. In the simplest algorithms, the time step interval is kept constant throughout the whole computational process. In most practical applications, a fixed time step leads to relatively inefficient solutions, and adaptive adjustments of $\Delta t$ are very desirable. In this section, an adaptive timestepping technique for thermo-viscoplastic structural analysis with damages is described.

For simplicity, consider the single ordinary differential equation,

$$
\dot{y}=f(y, t)
$$

The solution is advanced using a predictorcorrector scheme. The predictor phase consists of an Euler step:

$$
\begin{gathered}
y_{1+\Delta t}^{p}=y_{1}+\Delta t \dot{y}_{1} \\
\dot{y}_{1+\Delta}^{p}=f\left(y_{1+\Delta}^{p} t+\Delta t\right) .
\end{gathered}
$$
from

An error indicator $E[12,22]$ is then computed

$$
E=\frac{\left|\Delta t\left(\dot{y}_{1+\Delta}^{P}-\dot{y}_{1}\right)\right|}{2 y_{1+\Delta}^{P} \mid} .
$$

The error indicator is next compared with a preset error criterion and if the criterion is met, the timestep is small enough to proceed to the corrector stage. Otherwise, the predictor phase for eqs (18)-(21) is repeated with a smaller time step. On the other hand, if the error is smaller than the user-defined value $E_{\text {m. }}$, the time step is increased to improve computational efficiency. For the viscoplastic evolution equations with damage, the control variables used to calculate the error indicator were the components of a stress tensor $\sigma_{b}$, internal state variables $Z_{i}$, and the damage

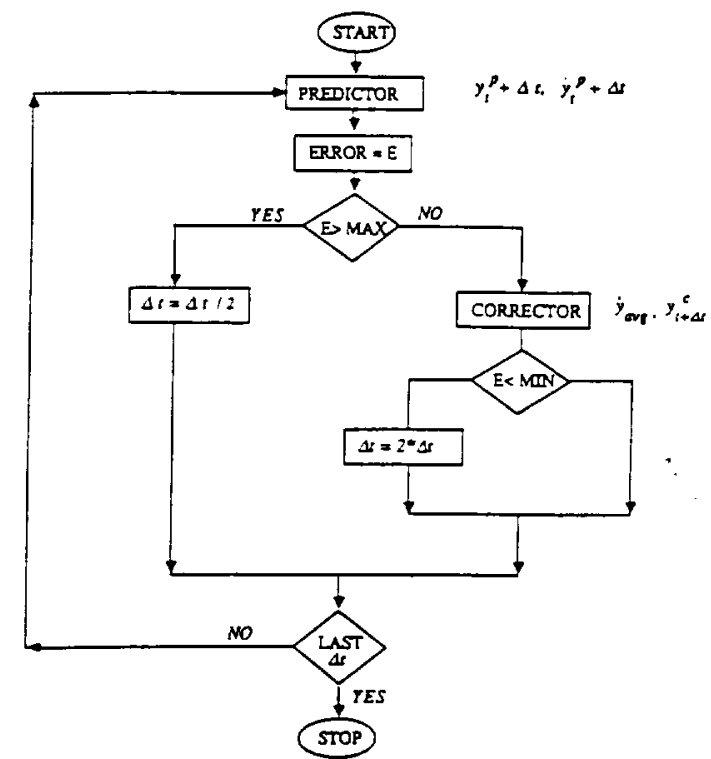

Fig. 2. Adaptive timestepping algorithm for viscoelastoplastic evolution problems.

variables $\omega_{1}$, with the maximum of these components selected as the controlling error. The corrector phase is the modified Newton scheme,

$$
\begin{aligned}
& \dot{y}_{\text {ave }}=\left(\dot{y}_{1}+\dot{y}_{1+\Delta}^{p}\right) / 2 \\
& \dot{y}_{i+\Delta}^{c}=y_{1}+\Delta t \dot{y}_{\text {ave }} .
\end{aligned}
$$

A flow chart depicting the adaptive scheme is shown in Fig. 2.

\section{NUMERICAL EXAMPLXS}

In this section, selected numerical examples oriented on verification of damage modeling and on illustration of advantages of adaptive methods in application to damage modeling are presentod. In particular, discussed are: (1) a simple tension creep test with experimental verification; (2) an isothermal structural problem with adaptive mesh refinement; (3) a thermostructural problem corresponding to the space shuttle main engine (SSME) combustion chamber liner.

\subsection{Tensile creep test}

The main purpose of this example is to verify the viscoplastic constitutive model and damage modeling by comparison of numerical predictions with available experimental data [17]. The example is a tensile creep test of nickel apperalloy B1900 + Hf subjected to constant stress level of $215 \mathrm{MPa}$ and temperature of $982^{\circ} \mathrm{C}$. For this case, both tertiary creep corresponding to continuum damage and final rupture of the specimen were observed. The resulting timestrain curves and damage histories are presented and compared with experimental data in Fig. 3. For comparison, the same examples were solved without 

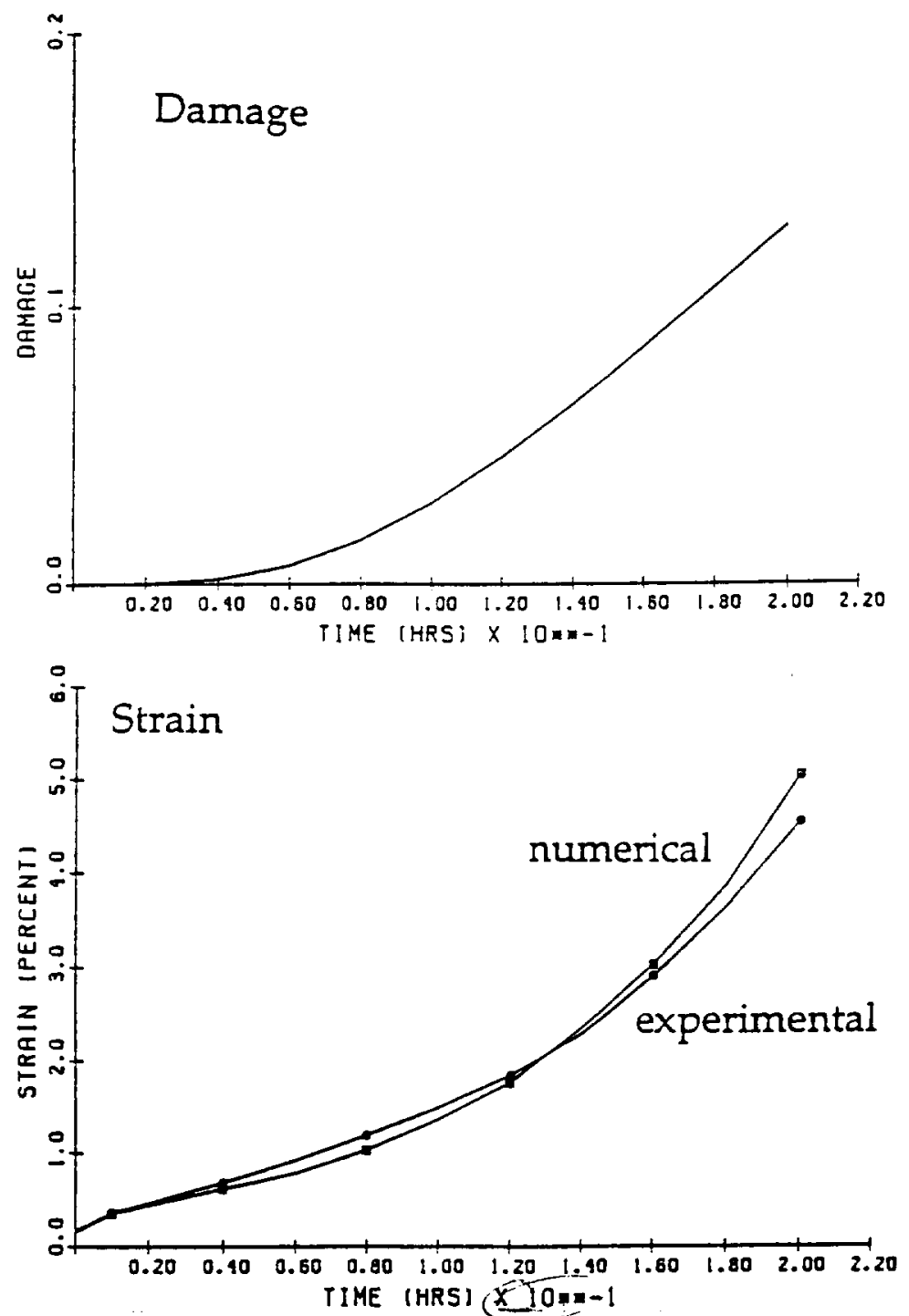

Fig. 3. Time histories of strain and damage for the creep tent at $982^{\circ} \mathrm{C}$ under atres of $215 \mathrm{MPa}$-experimental and numerical results.

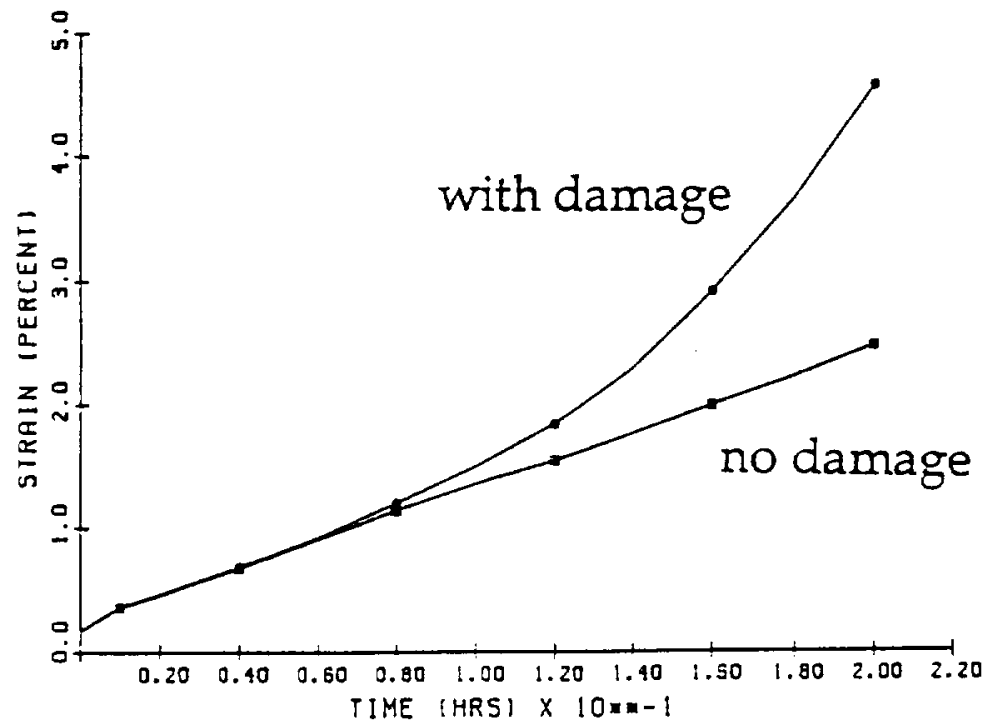

Fig. 4. Comparison of models with damage and without damage modeling. Time histories of strain for the creep test at $982^{\circ} \mathrm{C}$ under stress of $215 \mathrm{MPa}$. 
damage modeling. The results of these comparisons are presented in Fig. 4.

The following conclusions can be drawn from this example and other tests shown in Ref. [17]: (1) the damage evolution models describe correctly the tertiary crep zone and transition to rupture; (2) the evolution of damage and rupture time are extremely sensitive to the stress level-small variation of stress or material constants for damage cause considerable change in the rupture time. A more extensive study of this issue is presented in Ref. [17]; (3) the actual rupture of the experimental specimen occurs at damage levels smaller than the theoretical rupture condition $(\omega=1)$. This is most probably caused by the fact that local damage concentrations, say on material inhomogeneities, cause localized growth of continuum damage, transition to macrocracks, and their propagation before a uniform saturation of the whole cross-section with micro-cracks can occur; (4) without damage modeling, the tertiary creep zone and rupture of the material are not predicted by the viscoplastic constitutive theoriesthus damage modeling is necessary to capture these phenomena.

\subsection{L-shaped domain}

A plane stress $L$-shaped domain subjected to prescribed displacement rates is presented as an example of a mesh-adaptive isothermal analysis with damage modeling (Fig. 5). In particular, displacements on boundary segments indicated in Fig. 5 grow during $10 \mathrm{~s}$ to a value of -0.001 , and then are held steady

Material: B1900 + Hf

Temperature: $1000^{\circ} \mathrm{C}$

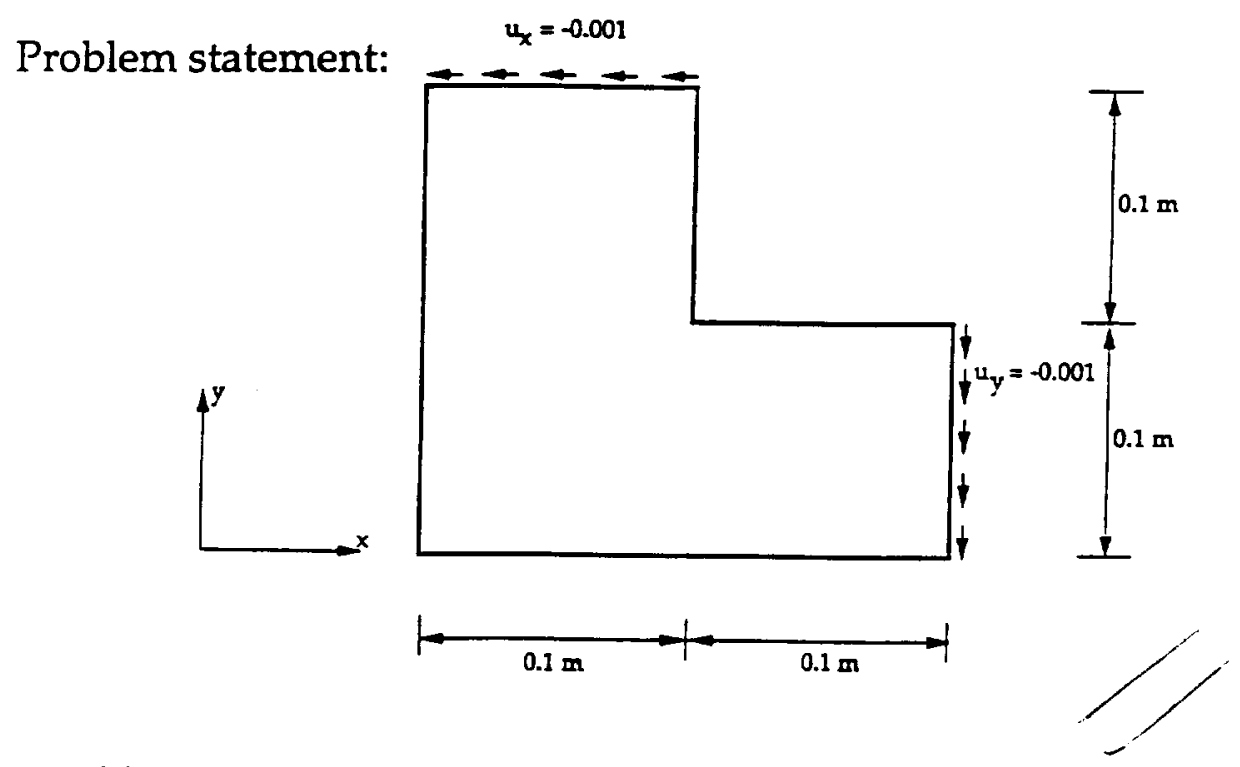

Load history:

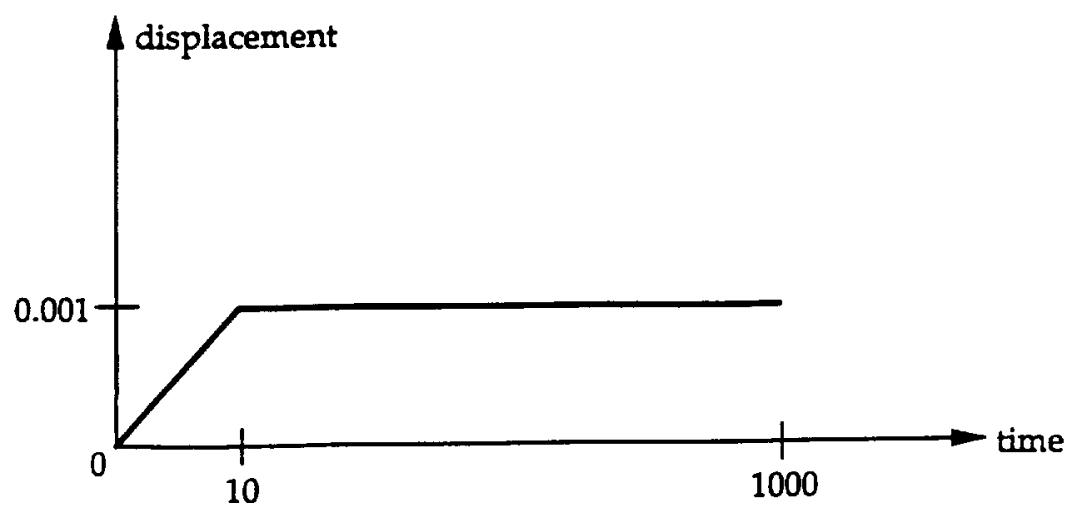

Fig. 5. Problem statement for an L-shaped domain. 


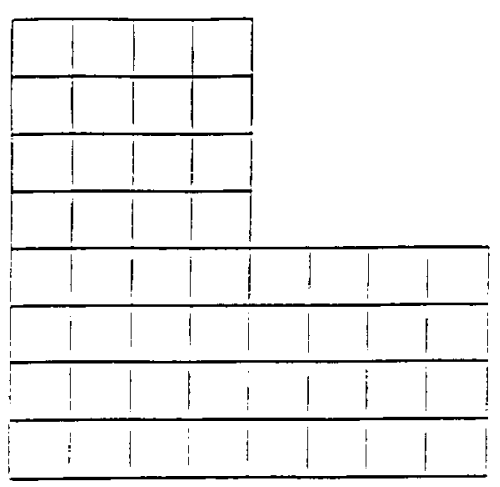

a) Initial Mesh

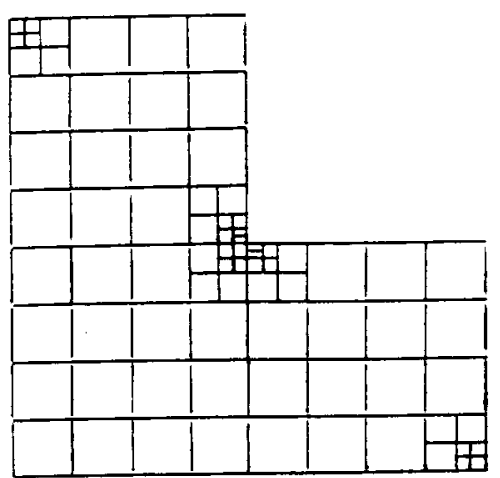

b) Adapted Mesh

Fig. 6. L-shaped domain. (a) initial mesh. (b) refined mesh.

until the final time of 1000 s. The temperature is equal $1000^{\circ} \mathrm{C}$, and the material is a superalloy $\mathrm{B} 1900+\mathrm{Hf}$. The problem was solved with adaptive mesh refinement. A comparison of initial mesh and the final adapted mesh is shown in Fig. 6. Contours of stress intensity and of damage are presented in Fig. 7. As expected, the adaptive procedure correctly refined the mesh around the corner singularities. The adapted mesh identified the maximum damage zone and predicted a high damage level, $\omega_{m}=0.45$. In fact, the damage zone is so concentrated that initiation of macro-crack can be predicted here. Although the location of crack initiation (corner of the $L$-shaped domain) is obvious in this test problem, in more general practical situations the appropriate adaptive procedure can also correctly identify such cases.

\subsection{SSME combustion chamber liner}

An adaptive analysis of an element of the SSME combustion chamber liner, subjected to thermal and mechanical loads has been taken as an illustration of the application of damage modeling to practical problems in aerospace structures. The problem for this example is illustrated in Fig. 8. The cross section of one cell of the linear is almost rectangular, with the internal coolant passage subjected to a pressure of

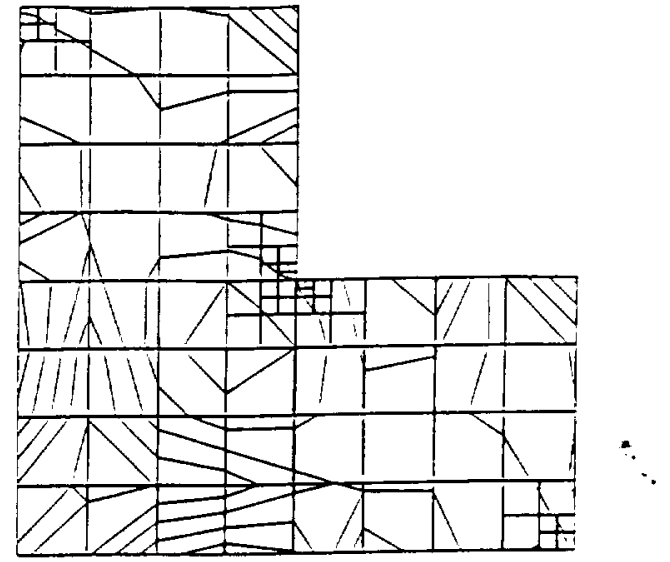

a)

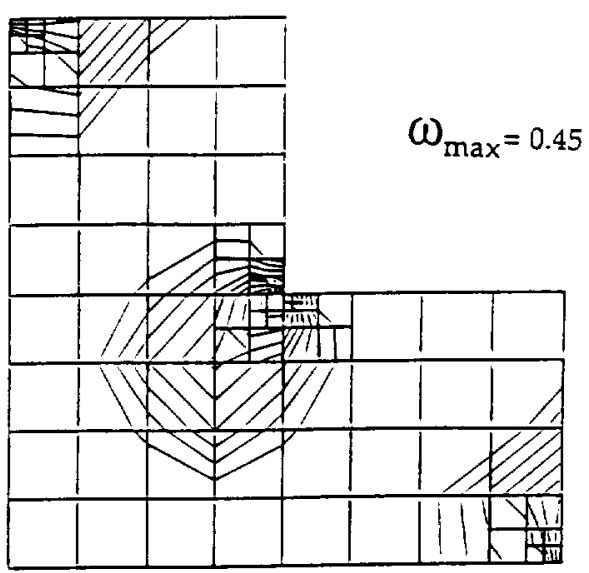

b)

Fig. 7. Effoctive stres contours (a) and damage level (b) for an L-shaped domain-refined mesh.

$3900 \mathrm{psi}(25.5 \mathrm{MPa})$. The initial temperature is of the order of $-200^{\circ} \mathrm{C}$, with almost uniform distribution throughout the cross-section. The thermal load history corresponds to a rapid heating of the liner from the inside in 58 up to a temperature close to $600^{\circ} \mathrm{C}$. This temperature gradient is held almost constant for $10 \mathrm{~s}$, and then the liner is cooled down in $5 \mathrm{~s}$. An idealization of this history is shown in Fig. 8.

The above problem was solved with boundary conditions, corresponding to a single cell of an axisymmetric liner, as shown in Fig. 9. The initial mesh for this problem is shown in Fig. 10. Starting from this mesh, a finite element adaptive procedure generated the refined mesh, as shown in Fig. 11. As will be shown later, this mesh correctly identifies regions of high stress and damage. For this problem, a combination of internal pressure and temperature gradient causes an extensive deformation of a bottom wall shown with exaggerated scale in Fig. 11. The corresponding temperature distribution is shown in Fig. 12 -high gradients are present near the bottom wall of the cell. The high temperature gradient and internal 
10

Geometry:

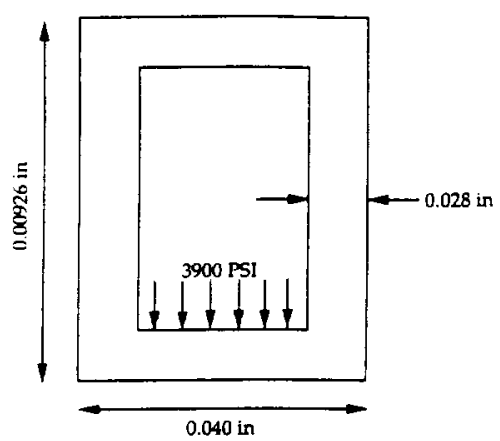

Material: copper

Thermal history:

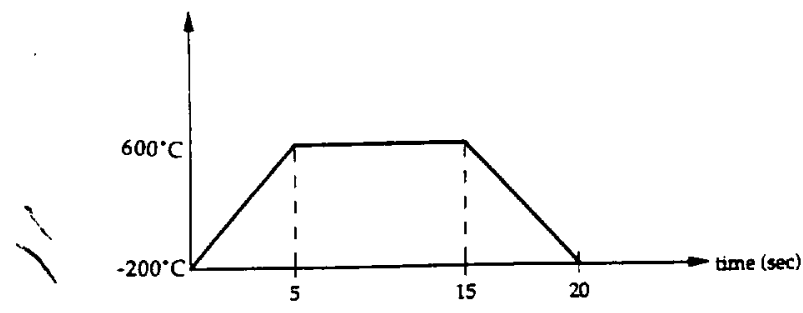

Fig. 8. SSME combustion chamber liner: problem statement and simplified thermal load history.

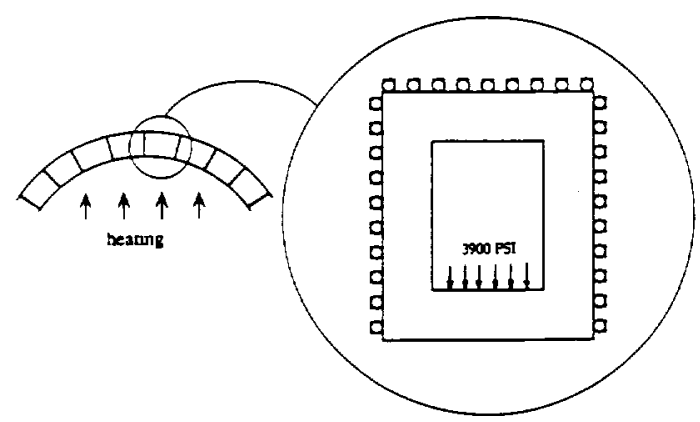

Fig. 9. Boundary conditions for the combustion chamber liner.

pressure cause high stress levels and some plastic deformation of the bottom wall as shown in Figs. 13 and 14. Due to the mostly compressive nature of stresses at this stage, damage development is very low and does not exceed $\omega \approx 10^{-7}$ at the end of the heating period.

During a cooling phase the situation is generally reversed-due to the specific boundary conditions and the plastic yield which occurred during beating, high tensile stresses develop in the bottom wall of the cell. The deformed configuration at the end of the cooling period is shown in Fig. 15. One can observe a thinning of the bottom wall caused by a combination of tensile stresses and internal pressure. The distribution of stresses at the end of the cooling period is shown in Fig. 16 and the corresponding principal plastic strain is illustrated in Fig. 17. In the cooling phase, the damage development is accelerated

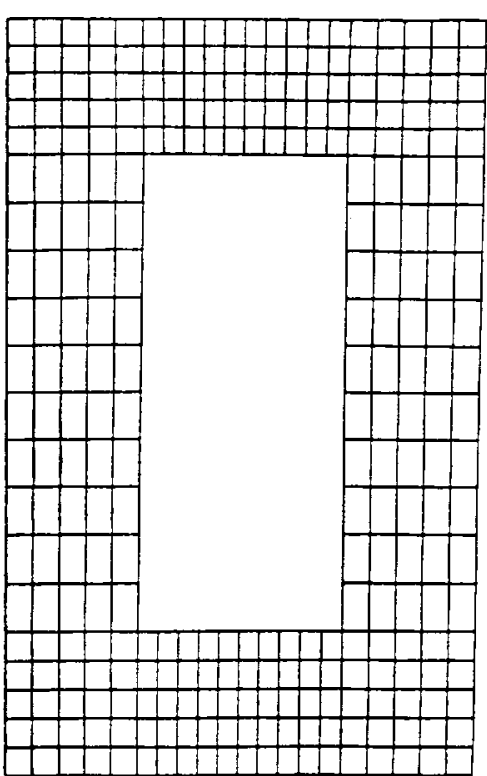

Fis. 10. SSME liner-initial mesh.

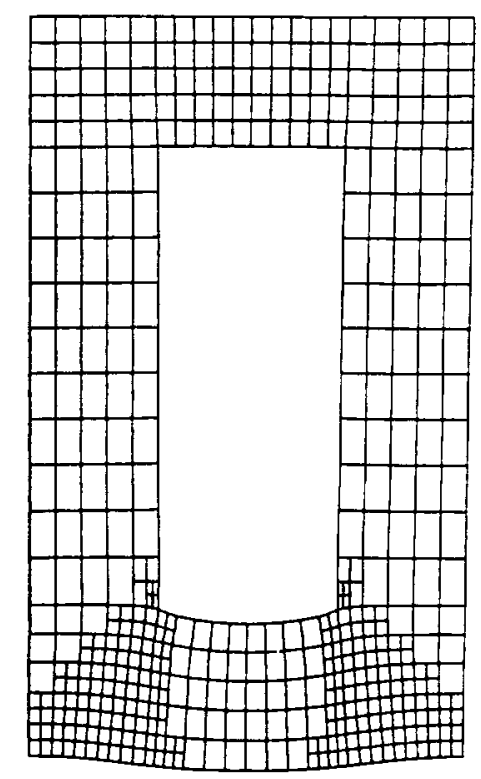

verome aris semet to.0000

Fig. 11. SSME liner-deformed configuration at the end of the beating period: $t=15$ ecconds.

in the bottom wall-the corresponding distribution of damage is shown in Fig. 18.

To better illustrate the development of the damage pattern, histories of damage for the inner lower corner and for the center of lower face of the cell are shown in Fig. 19. It can be seen that damage development is indeed accelerated during the cooling phase. One can expect that further damage will evolve in the consecutive cycles of heating-cooling. This example clearly illustrates the potential of adaptive finite 


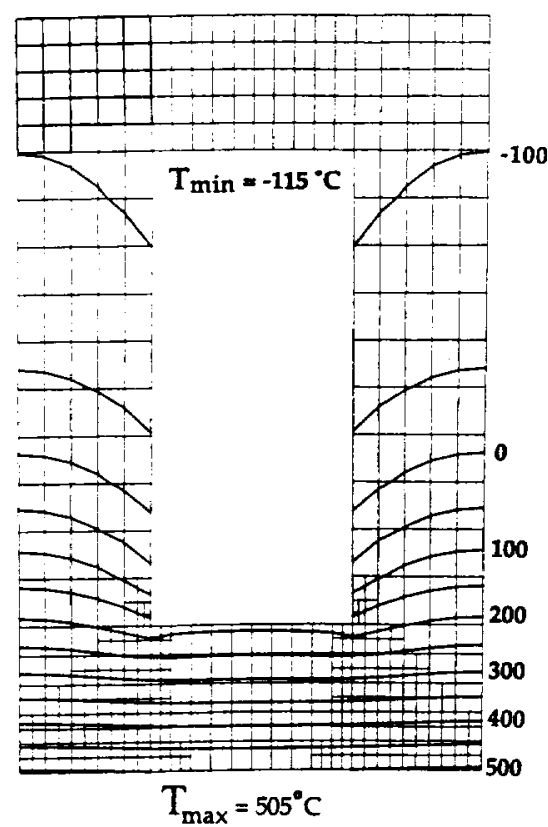

Fig. 12. SSME liner-distribution of temperatures at the end of the heating period: $t=15$ reconds.

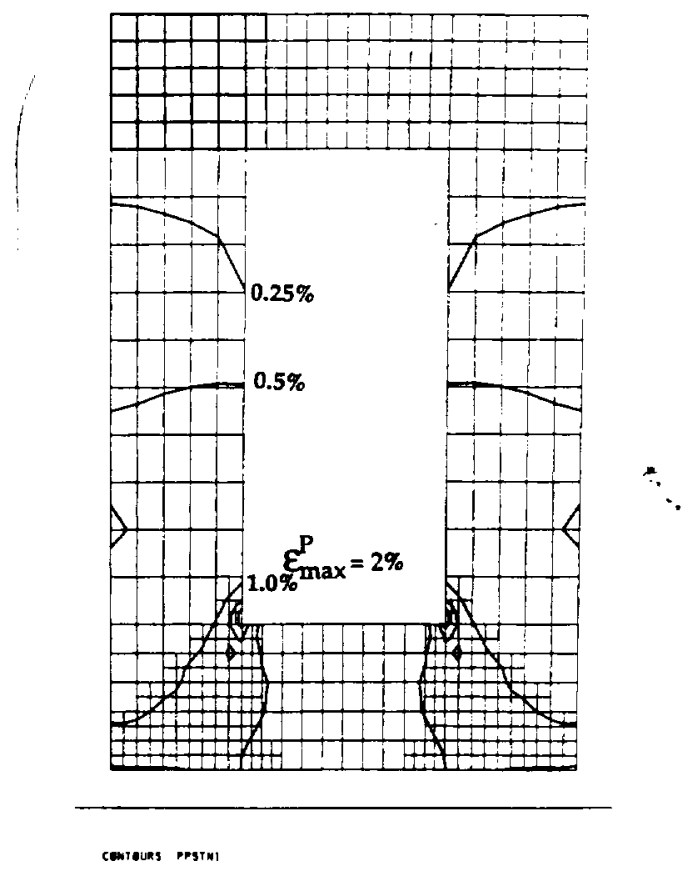

Fig. 14. SSME liner-contours of maximum plastic strain at the end of the heating period: $t=15$ soconds.
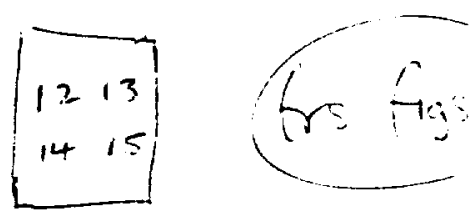

$$
\sigma_{\max }^{(i)}=110 \mathrm{MPa} \quad(16 \mathrm{KSI})
$$

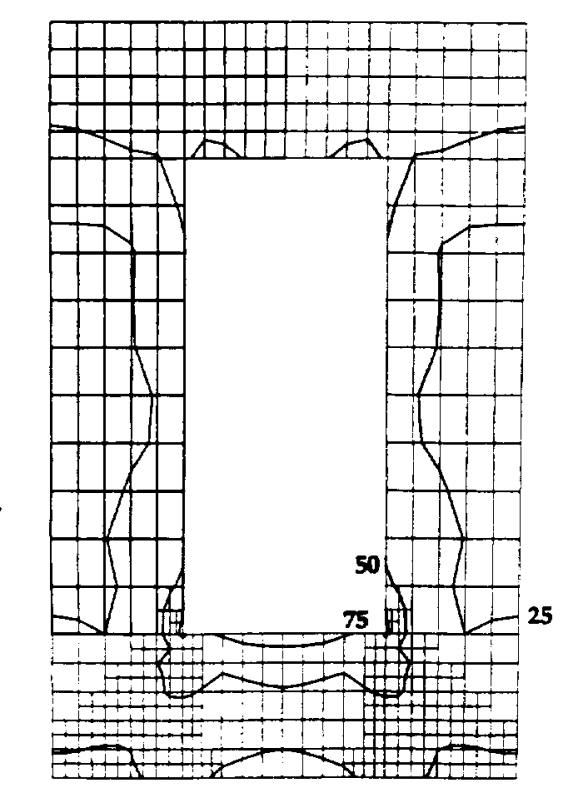

-oniguns stas

Fig. 13. SSME liner-distribution of stress intensity at the end of the heating period: $t=15$ seconds.

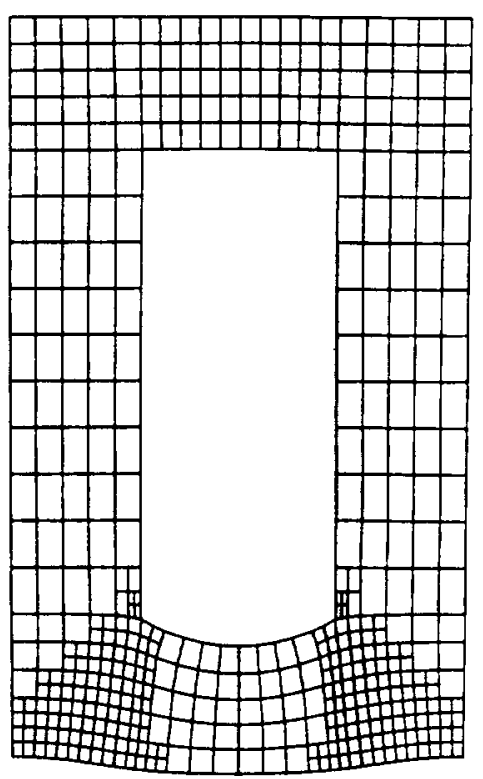

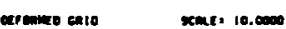

Fig. 15. SSME liner-deformed configuration at the end of the cycle: $t=20$ seconds. 


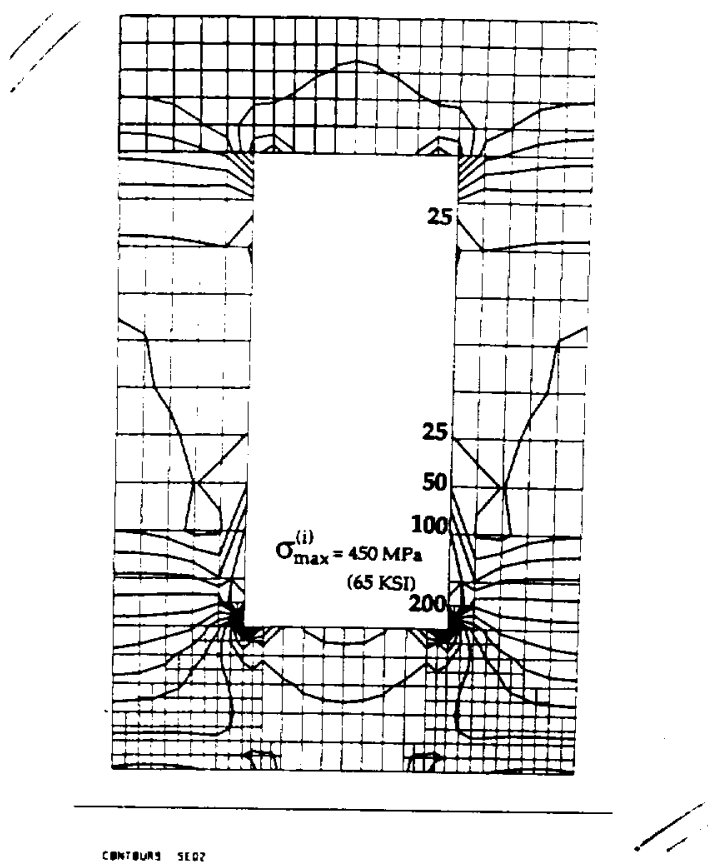

Fig. 16. SSME liner-distribution of stress intensity at the end of the cycle: $t=20$ seconds.

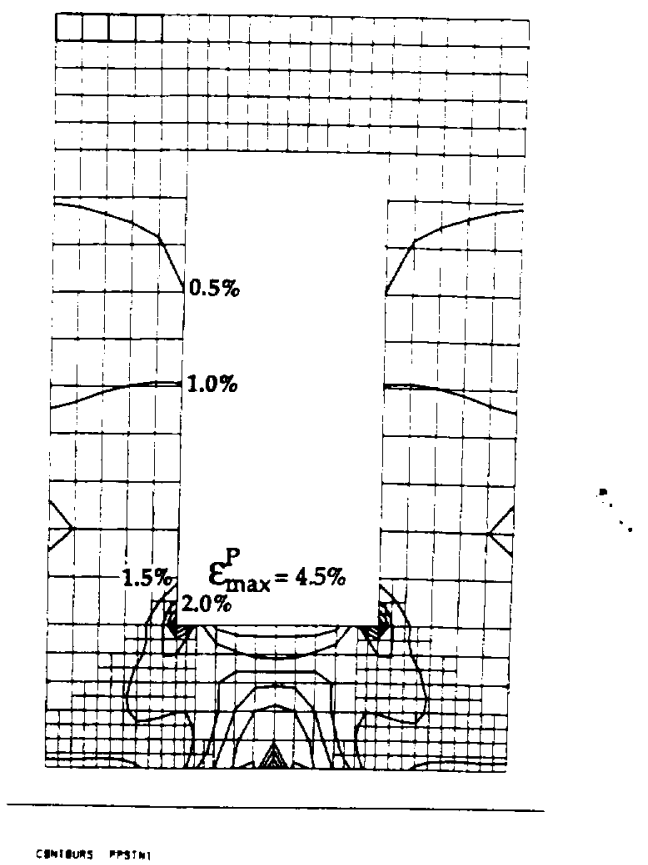

Fig. 17. SSME liner-distribution of principal plastic strain at the end of the cycle: $t=20$ seconds.

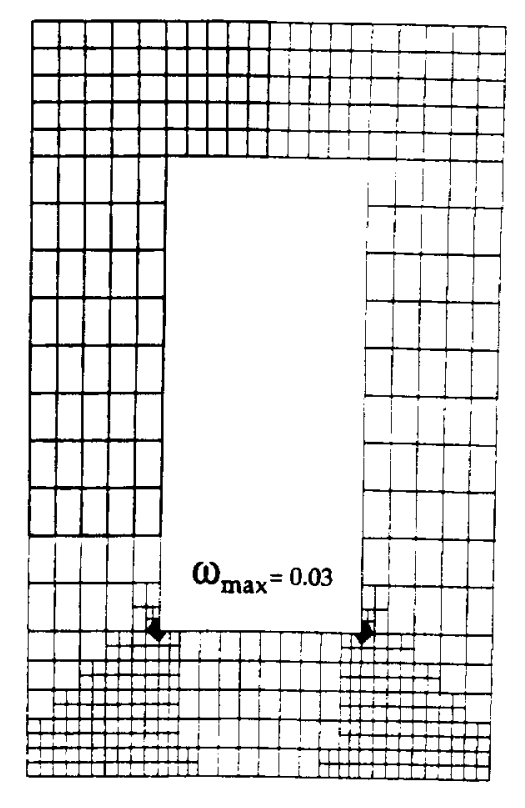

comrouns amance

Fig. 18. SSME liner-contours of damage at the end of the cycle: $t=20$ seconds. 


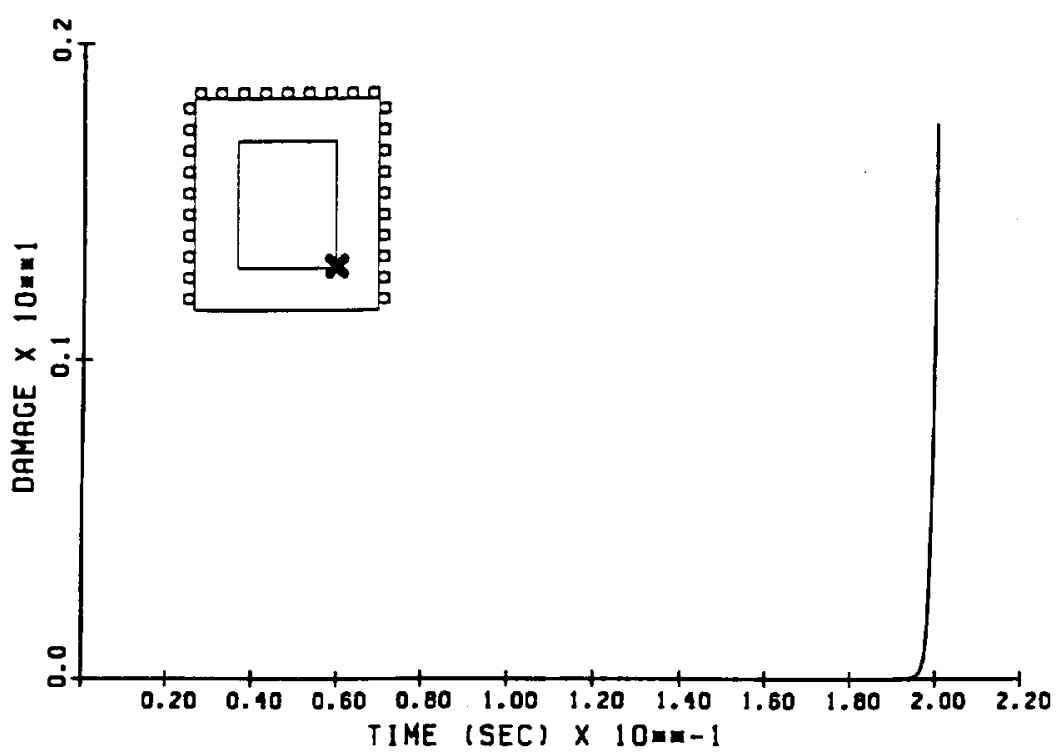

a)

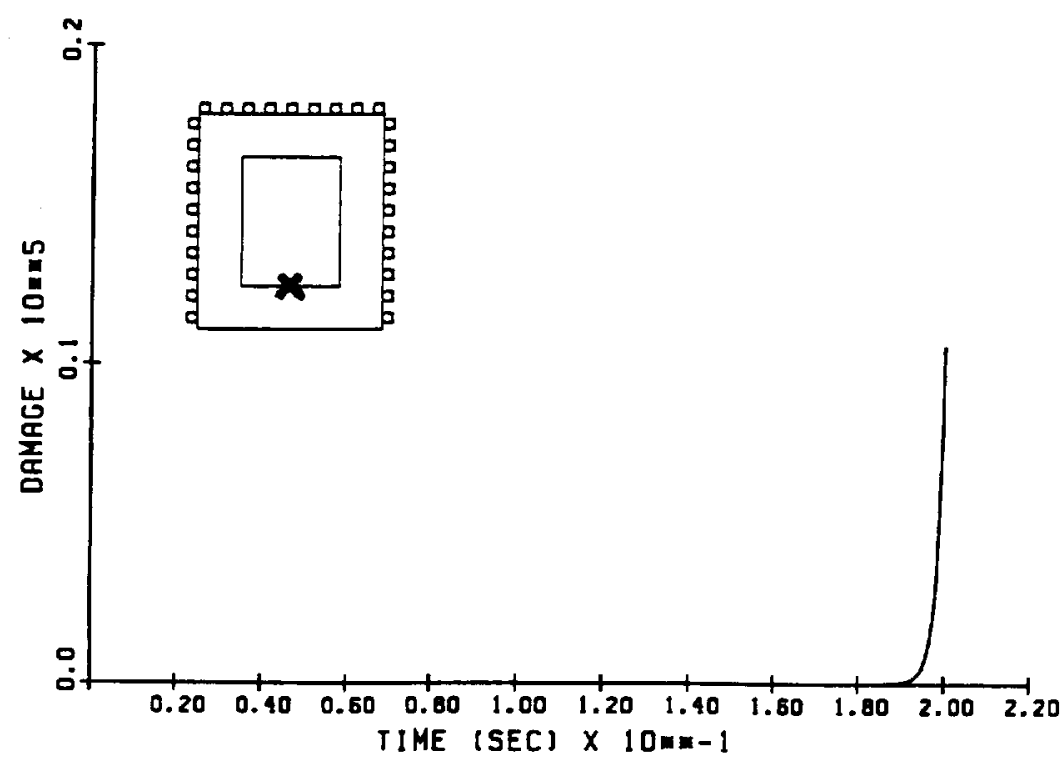

b)

Fig. 19. SSME liner-histories of damage at various locations in a crose-section. Note a different scale for each plot.

element methods in the identification of critical zones and damage patterns of aerospace structures.

\section{CONCLUSIONS}

The formulations and results presented in this paper clearly confirm the feasibility of developing adaptive finite element methods for modeling damage evolution and for making life predictions of hightemperature structures.
The error estimation and adaptive mesh refinement not only provide accuracy control and an optimal solution at a minimum cost, but are also capable of pinpointing locations, where concentrated continuum damage growth leads to initiation of macro-cracks.

Similarly, the adaptive time-stepping guarantees error control and optimal adjustments of time steps, with the actual values of the time steps often varying by the factors of 1000 depending on the evolution of the nonlinear deformation in the structure. 
As far as damage modeling is concerned, the following remarks are applicable: (1) while theoretical formulations of the damage modeling are presently quite mature, the number of reliable experimental studies dedicated to the determination of material constants and the verification of theory is still very limited. (2) The rate of continuum damage development is very sensitive to the temperature and the stress level-a small variation of these parameters leads to considerable changes in the predicted time to rupture. This sensitivity emphasizes the importance of an accurate prediction of the stresses by the application of error estimation and mesh refinement. (3) This paper addressed only the low-cycle damage development, wherein rupture happens after a relatively short time, and the damage parameter can be effectively integrated as a function of time. (4) For many practical applications it is also important to consider high-cycle damage, wherein it is much more efficient to integrate damage over the number of cycies, rather than directly over time. The extrapolation techniques are useful in cases of long-term damage evolutions.

Acknowledgement-Helpful discussions for the Space Shuttle Main Engine (SSME) combustor liner problem with John Price Jr/NASA-MSFC are gratefully acknowlodged. The second and third authors were supported by the NASAMarshall Space Flight Center under Contract NAS8-39311.

\section{DDFERENCTS}

1. S. R. Bodnet and Y. Partom, Constitutive equations for elastio-viscoplastic strain-hardening materials. J. appl. Mech. 12, 385-389 (1975).

2. A. K. Miller (Ed.), Unified Constitutive Equations for Creep and Plasticily. Elsevier, Oxfond (1987).

3. K. S. Chan, U. S. Lindholm, S. R. Bodner, J. R. Hill, R. M. Weber and T. O. Meyer, Constitutive modeling for isotropic materials (HOST). Third Annual Status Report, NASA CR-179522, Southwest Research Institute, San Antonio, TX, June (1986).

4. K. S. Chan, U. S. Lindholm and S. R Bodner, Constitutive modeling for isotropic materials (HOST). Final Report, NASA CR-182132, Southwest Research Institute, San Antonio, TX, June (1988).

5. A. D. Freed, Thermovircoplastic model with application to copper. NASA-TP 2845, December (1988).

6. A. D. Freed and M. J. Verilli, A viscoplastic theory applied to copper. NASA-TM 100831, September (1988).

7. A. D. Freed and K. P. Walker, Refinements in a viecoplastic model. NASA-TM 102338, December (1989).
8. M. Newman, Z. Zaphir and S. R. Bodner, Finite element analysis for time-dependent inelastic material behavior. Comput. Struct. 6, 157-162 (1976).

9. A. Kaufman, J. H. Laflen and U. S. Lindholm, Unified constitutive material models for nonlinear finito-element structural analysis. AIAA/SAE/ASME/ASE 2lst Joint Propulsion Conf., AIAA Paper no. 85-1418, Monterey, CA, 8-10 July (1985).

10. V. Moreno and E. H. Jordan, Prediction of material thermomechanical response with a unified viscoplastic constitutive model. Int. J. Plast. 2, 224-245 (1986).

11. V. K. Arya, Nonlinear structural analysis of cylindrical thruse chambers using viscoplastic model. J. Propulsion Power 8, 564-598 (1992).

12. J. M. Bass and J. T. Oden, Adaptive finite element methods for a class of evolution problems in viscophaticity. Int. J. Engng. Sci. 25, 623-653 (1987).

13. E. A. Thorton, J. T. Oden, W. W. Tworzydlo and S. K. Youn, Thermoviscoplastic analysis of hypersonic structures aubjected to severe serodynamic heating. J. Aircraft 27, 826-836 (1990)

14. M. F. Ashby and M. Raj, Proc. of Conf. Mechanics and Physics of the Metals Society, Cambridge, Institute of Physics (1974).

15. G. Greenwood, Proc. Int. Cong. on Metals, Cambridge, Vol. 2 pp. 29-105 (1973).

16. L. M. Kechanov, Time of the rupture process under creep conditions. Irv. Akad. Nouks SSR Otd. Tech. Naue. 26-31 (1958).

17. S. R. Bodner and K. S. Chan, Modeling of continuum damage for application in elast-viscoplastic constitutive equations. Engng Fract. Mech. 25, 705-712 (1986).

18. F. A. Lockie and D. R. Hayhurst, Constitutive equations for creep rupture. Acta. Metal. 25, 1059-1070 (1977).

19. J. C. Simo, On a fully three-dimensional finite atrain viscoplastic damage model: formulation and computational sepects. Comput. Meth. appl. Mech. Engng 60, 153-173 (1987).

20. R. S. Neleon, Application of cyclic demage socumulation Ife prodiction model to high temperature components. Proc. Conf. Structural Durability, Cleveland, OH (1989).

21. V. Kumar, M. Morjaria and S. Mukherjoc, Numerical integration of some constitutive models of inelastic do formation. J. Engng Mater. Technol. 102, 92-96 (1980).

22 L. Demkowicz, J. T. Oden, W. Rachowicz and O. Hardy. Toward a univeral $h-p$ adaptive finite element ctratey, Part I: constrained approximation and data tructure. Compur. Meth. appl. Mech. Engng 77, 79-112 (1989).

23. J. T. Oden and L. Demkowicz, Advances in adaptive improvements: a sudy of adaptive finite element methods in computational mochanics. In State of the Art Suroeys in Computational Mechanics (Edited by A. K. Noor and J. T. Oden) ASME, New York (1986).

24. W. Rachowicz, J. T. Oden and L. Demkowicz, Toward a univerel $h \rightarrow p$ adoptive finite element strategy, Part III: Design of $h-p$ meshea. Comput. Meth. appl. Mech. Engng 7, 181-212 (1989). 